\title{
Factores asociados al estado nutricional en pacientes adultos mayores hospitalizados
}

\author{
Factors associated with nutritional status in \\ hospitalized elderly patients
}

\author{
Diego Andrés Chavarro-Carvajal, Miguel Germán Borda, \\ Nicolás Núñez, Daniel Cortés, Santiago Sánchez, Nicolás Ramírez \\ - Bogotá, D.C. (Colombia)
}

\section{Resumen}

Introducción: la desnutrición es una condición frecuente en las personas mayores de 65 años. El mal estado nutricional se ha relacionado con mayores estancias hospitalarias, complicaciones, aumento en comorbilidades y mortalidad. Este estudio busca describir la relación entre el estado nutricional de adultos mayores hospitalizados y factores como red de apoyo, funcionalidad y presencia de demencia.

Material y métodos: se llevó a cabo un estudio descriptivo de corte transversal analítico basado en la revisión de historias de los pacientes hospitalizados por el Servicio de Geriatría del Hospital Universitario San Ignacio de Bogotá D.C. La variable dependiente fue el estado nutricional medido con el instrumento Mini-Nutritional Assessment y se usaron como variables independientes factores sociodemográficos, el estado funcional, el diagnóstico previo de demencia, la red de apoyo, delirium, tiempo de estancia hospitalaria, comorbilidades.

Resultados: se incluyeron 887 pacientes con edad promedio de 85.43 años, de los cuales $43.07 \%$ eran hombres. En el modelo de regresión logística la presencia de delirium OR 2.27 (IC 1.48-3.48), el diagnóstico previo de demencia OR 2.48 (IC 1.63-3.77) y el mayor tiempo de estancia hospitalaria OR 1.05 (IC 1.0-1.10 ) tuvieron una asociación de aumento de riesgo con desnutrición.

Conclusión: se encontró una asociación significativa entre mal estado nutricional con menor funcionalidad, mayor estancia hospitalaria, tener diagnóstico de demencia, presentar delirium, tener un mayor número de comorbilidad y mayor mortalidad. Se requieren más estudios que aborden este tema. (Acta Med Colomb 2018; 43: 69-73).

Palabras clave: desnutrición, estado nutricional, adulto mayor, hospitalización.

\section{Abstract}

Introduction: malnutrition is a prevalent condition in people older than 65 years. Poor nutritional status has been associated to longer hospital stays, complications, increased comorbidities and mortality. This study aims to describe the relationship between the nutritional status of hospitalized older adults and factors such as support network, functionality and the presence of dementia.

Materials and methods: a descriptive cross-sectional analytical study was carried out based on the revision of medical records of patients hospitalized by the Geriatrics Unit at San Ignacio University Hospital in Bogota, Colombia. Nutritional status measured through theMini-Nutritional Assessment was considered to be the dependent variable. Sociodemographic factors, functional status, previous diagnosis of dementia, support network, delirium, length of hospital stay and presence of comorbidities were used as independent variables.

Results: 887 patients with an average age of 85.43 were included. $43.07 \%$ were men. The logistic regression model revealed that the presence of delirium OR 2.27 (CI 1.48-3.48), the diagnosis of dementia OR 2.48 (CI 1.63-3.77) and the longer hospital stay OR 1.05 (CI 1.0-1.10) had an association of increased risk with malnutrition.
Dr. Diego Andrés Chavarro-Carvajal: Especialista en Medicina Interna y Geriatría, Magister Epidemiología, Candidato a Doctor en Investigación en Gerontología. Profesor del Instituto de Envejecimiento, Facultad de Medicina, Pontificia Universidad Javeriana. Médico Geriatra Unidad de Geriatría, Hospital Universitario San Ignacio; Dr. Miguel Germán Borda: Investigador del Centre for Age-Related Diseases, Stavanger University Hospital, Stavanger, Norway. Miembro del Semillero de Neurociencias y Envejecimiento, Instituto de Envejecimiento, Facultad de Medicina, Pontificia Universidad Javeriana; Nicolás Núñez Ordóñez, Daniel Cortés Sarmiento, Santiago Sánchez-Villalobos y Nicolás Ramírez Rozo: Estudiantes de Medicina. Miembros del Semillero de Neurociencias y Envejecimiento. Instituto de envejecimiento, Facultad de Medicina, Pontificia Universidad Javeriana. Bogotá, D.C. (Colombia).

Correspondencia: Dr. Diego Andrés Chavarro, Bogotá, D.C. (Colombia).

E-mail: diegoandreschavarro@gmail.com Recibido: 25/IV/2017 Aceptado: 10/IV/2018 
Conclusion: a significant association was found between poor nutritional status with decreased functionality, longer hospital stays, having a diagnosis of dementia, presenting delirium, having a greater number of comorbidity and higher mortality. Future studies addressing this issue are required. (Acta Med Colomb 2018; 43: 69-73). (Acta Med Colomb 2018; 43: 69-73).

Keywords: malnutrition, nutritional status, elderly, hospitalization.

\section{Introducción}

La desnutrición es una condición frecuente en las personas mayores de 65 años. Varios estudios estiman un riesgo mayor a $40 \%$ a presentar desnutrición en pacientes mayores hospitalizados $(1,2)$, el cual tiende a aumentar con la edad logrando incluso a duplicarse en la octava década de la vida (3). La desnutrición en pacientes adultos mayores está directamente relacionada con la presencia de síntomas depresivos $(4,5)$, una mayor estancia hospitalaria, mayores complicaciones durante la hospitalización y el aumento significativo de comorbilidades, peor función cognitiva, atrofia cerebral, sarcopenia, alteración funcional y mortalidad $(2,4,6,7,9)$.

Adicionalmente, diversos estudios demuestran que la prevalencia de demencias en la población adulta mayor va en aumento hacia los próximos años, hallazgo de gran importancia debido a que su presencia aumenta considerablemente el riesgo nutricional en dichos pacientes (3).

Uno de los principales determinantes de desnutrición en la población adulta es el aislamiento social que tiende a aumentar hasta 2.5 veces el riesgo de presentar desórdenes alimenticios (1) y está estrechamente relacionado con la presencia de trastornos depresivos (5). Desde el punto de vista de los sistemas de salud, diversos estudios afirman que, al realizar una valoración nutricional adecuada, los costos hospitalarios disminuyen considerablemente, siendo más relevante en la población mayor a 55 años a pesar de su asociación con un mayor número de comorbilidades (11).

El objetivo de este estudio es describir la relación entre el estado nutricional de pacientes mayores hospitalizados y variables como la red de apoyo, la funcionalidad y la presencia de demencia.

\section{Material y métodos}

Se llevó a cabo un estudio descriptivo de corte transversal cuantitativo usando los registros de la base de datos de pacientes hospitalizados por el Servicio de Geriatría del Hospital Universitario San Ignacio (HUSI) de Bogotá, D.C. para el periodo comprendido entre el $1^{\circ}$. de enero de 2014 hasta el 30 de abril de 2016.

Se incluyeron un total de 887 pacientes luego de excluir 132 registros de los análisis estadísticos al no contarse con los datos completos necesarios para el análisis de los datos.

La información registrada incluyó variables sociodemográficas como nombre, edad, sexo, fecha de ingreso y egreso de la hospitalización, tiempo de estancia hospita- laria (TEH) y reingreso. Además, variables como estado funcional, estado mental (demencia), redes de apoyo social, presencia de comorbilidades y aparición de síndrome confusional agudo (delirium) durante la hospitalización.

La variable dependiente se usó en el estado nutricional como una variable cualitativa ordinal, evaluado por medio del Mini-Nutritional Assessment ${ }^{\circledR}$ (MNA) (12). Los resultados fueron clasificados en dos grupos para el análisis estadístico: "normal" (24-30 puntos en el MNA) y "desnutrición", que incluía a la población clasificada en las categorías de riesgo de malnutrición y malnutrición según el puntaje del MNA (0-23.5 puntos). Para la valoración del estado funcional se utilizó el resultado de la escala de Barthel (13), medida al ingreso y al egreso. En cuanto al estado mental se consideró el diagnóstico previo de demencia. Las redes de apoyo fueron evaluadas como parte de la valoración geriátrica integral, clasificándose en cuatro categorías: buena red de apoyo, riesgo social, mala red de apoyo y paciente institucionalizado.

Este estudio fue aprobado por el comité de ética e investigación de la Pontificia Universidad Javeriana y el Hospital Universitario San Ignacio.

El análisis estadístico de los datos se realizó por medio del paquete estadístico STATA 12. Para las variables cuantitativas se calcularon promedios y desviaciones estándar. Se utilizó el análisis univariado para explorar los valores extremos y la distribución normal para ajustar y categorizar las variables. Las variables categóricas se expresan como frecuencias y porcentajes, mientras que las medias y las desviaciones estándar se utilizaron para las variables continuas. Se analizó la asociación entre las dependientes y las independientes categóricas, utilizando pruebas de chi-cuadrado para las variables categóricas y t-test para las continuas. Se realizó un modelo de regresión logística multivariada en el cual la variable resultado fue desnutrición y considerando como variables independientes edad, sexo, puntaje de la escala de Barthel, presencia de demencia, red de apoyo social, presencia de delirium, TEH y comorbilidades, las cuales se incluyeron dentro del modelo en orden ascendente según significancia clínica y estadística.

\section{Resultados}

La edad promedio fue de $85.43( \pm 7.02)$ con una proporción de hombres del $43.07 \%(\mathrm{n}=382)$, demencia de $71.25 \%(\mathrm{n}=632)$ y delirium del $55.13 \%(\mathrm{n}=489)$ y finalmente una mortalidad intrahospitalaria del $10.48 \%$ $(n=93)$. Setecientos sesenta y cinco pacientes fueron 
clasificados como poseedores de una buena red de apoyo, mientras que 72 y 50 tenían una red regular o mala respectivamente. Además, el Barthel promedio al ingreso fue de $45.99( \pm 34.46)$ y la estancia hospitalaria promedio fue alrededor de una semana; $7.16( \pm 7.12)$ (Tabla 1).

En adición, se encontró que las personas con malnutrición tienen una mayor prevalencia de delirium $(57.45 \%$ vs $37.25 \%$ ), demencia ( $73.63 \%$ vs $52.94 \%$ ) y un peor estado funcional según la escala de Barthel (43.46 ( \pm 33.07$)$ vs $65.49( \pm 33.85))$. Además, presentan una mayor mortalidad (11.48 vs $2.94 \%)$, número de comorbilidades (5.18 $( \pm 2.66)$ vs $4.54( \pm 2.20))$ y días de estancia hospitalaria $(7.3( \pm 7.32)$ vs $5.6( \pm 5.01))$. El valor p para estas variables fue $<0.05$ (Tabla 2).

Además, en el modelo de regresión logística multivariada se encontraron como factores independientes asociados a desnutrición la presencia de delirium con OR 2.27 (IC 1.48-3.48, p= 0.00), diagnóstico previo de demencia con un OR 2.48 (IC 1.63-3.77, p=0.00), el mayor tiempo de estancia hospitalaria con OR 1.05 (IC $1.008-1.100, \mathrm{p}=0.018$ ), $\mathrm{y}$ una mayor funcionalidad medida con el Barthel al ingreso con un OR=0.98 (IC 0.97-0.987, $\mathrm{p}=0.00$ ) (Tabla 3).
Tabla 1. Características sociodemográficas.

\begin{tabular}{|c|c|}
\hline Características & Promedio (DE) o n (\%) \\
\hline Edad & $85.43(7.02)$ \\
\hline \multicolumn{2}{|l|}{ Sexo } \\
\hline Hombre & $382(43.07)$ \\
\hline Mujer & $505(56.93)$ \\
\hline Días de estancia hospitalaria (0-66) & $7.16(7.12)$ \\
\hline Barthel ingreso $(0-100)$ & $45.99(34.46)$ \\
\hline \multicolumn{2}{|l|}{ Demencia } \\
\hline No & $255(28.75)$ \\
\hline Sí & $632(71.25)$ \\
\hline \multicolumn{2}{|l|}{ Red de apoyo } \\
\hline Buena & $765(86.25)$ \\
\hline Regular & $72(8.12)$ \\
\hline Mala & $50(5.64)$ \\
\hline Comorbilidades (0-19) & $5.11(2.62)$ \\
\hline \multicolumn{2}{|l|}{ Delirium } \\
\hline No & $398(44.87)$ \\
\hline Sí & $489(55.13)$ \\
\hline \multicolumn{2}{|l|}{ Mortalidad } \\
\hline Sí & $93(10.48)$ \\
\hline No & $794(89.52)$ \\
\hline
\end{tabular}

Tabla 2. Análisis bivariado, estado nutricional y variables independientes ( $n=887$ ).

\begin{tabular}{|c|c|c|c|}
\hline Variable & $\begin{array}{c}\text { Normal } \\
\mathbf{n}=102(11.5 \%) \\
\mathbf{n}(\%) \text { o media(DE) }\end{array}$ & $\begin{array}{c}\text { Malnutrición } \\
\text { n=785 (88.5\%) } \\
\text { n(\%) o media(DE) }\end{array}$ & $\mathbf{p}$ \\
\hline Edad (56-102) & 84.25 (7.6) & $85.58(6.66)$ & 0.0628 \\
\hline \multicolumn{4}{|l|}{ Sexo } \\
\hline Hombre & $42(41.18 \%)$ & $340(43.41 \%)$ & \multirow[t]{2}{*}{0.682} \\
\hline Mujer & $60(58.82 \%)$ & 445 (56.69\%) & \\
\hline Días de estancia hospitalaria (0-66) & $5.6(501)$ & $7.3(7.32)$ & 0.018 \\
\hline Barthel ingreso (0-100) & $65.49(33.07)$ & $43.46(33.85)$ & $<0.0001$ \\
\hline \multicolumn{4}{|l|}{ Demencia } \\
\hline No & $48(47.06 \%)$ & $207(26.37 \%)$ & \multirow[t]{2}{*}{$<0.001$} \\
\hline Sí & $54(52.94 \%)$ & $578(73.63 \%)$ & \\
\hline \multicolumn{4}{|l|}{ Red de apoyo } \\
\hline Buena & $93(91.18 \%)$ & $672(85.61 \%)$ & \multirow[t]{3}{*}{0.361} \\
\hline Regular & $6(5.88 \%)$ & $66(8.41 \%)$ & \\
\hline Mala & $3(2.94 \%)$ & $47(5.64 \%)$ & \\
\hline Comorbilidades (0-19) & $4.54(2.20)$ & $5.18(2.66)$ & $<0.021$ \\
\hline \multicolumn{4}{|l|}{ Delirium } \\
\hline No & $64(62.75 \%)$ & $334(42.55 \%)$ & \multirow[t]{2}{*}{$<0.0001$} \\
\hline Sí & $38(37.25 \%)$ & $451(57.45 \%)$ & \\
\hline \multicolumn{4}{|l|}{ Mortalidad } \\
\hline Sí & $3(2.94 \%)$ & $90(11.48 \%)$ & \multirow[t]{2}{*}{$<0.001$} \\
\hline No & $99(97.06 \%)$ & $695(88.54 \%)$ & \\
\hline
\end{tabular}


Tabla 3. Análisis multivariado desnutrición y factores asociados.

\begin{tabular}{|l|c|c|}
\hline Variable & OR (IC) & p \\
\hline Edad (56-102) & $1.02(0.99-1.05)$ & 0.063 \\
\hline Sexo & $0.91(0.60-1.39)$ & 0.0682 \\
\hline $\begin{array}{l}\text { Barthel (continua } \\
\text { 0-100) }\end{array}$ & $0.98(0.97-0.987)$ & $<0.0001$ \\
\hline $\begin{array}{l}\text { Tiempo de estancia } \\
\text { hospitalaria } \\
\text { (continua) }\end{array}$ & $1.05(1.008-1.100)$ & 0.018 \\
\hline $\begin{array}{l}\text { Demencia } \\
\text { (tener demencia) }\end{array}$ & $2.48(1.63-3.77)$ & $<0.0001$ \\
\hline $\begin{array}{l}\text { Red de apoyo } \\
\text { (mala red de apoyo) }\end{array}$ & $1.73(0.85-3.54)$ & 0.129 \\
\hline $\begin{array}{l}\text { Comorbilidades } \\
\text { (continua) }\end{array}$ & $1.10(1.01-1.21)$ & 0.022 \\
\hline $\begin{array}{l}\text { Delirium } \\
\text { (tener delirium) }\end{array}$ & $2.27(1.48-3.48)$ & $<0.0001$ \\
\hline
\end{tabular}

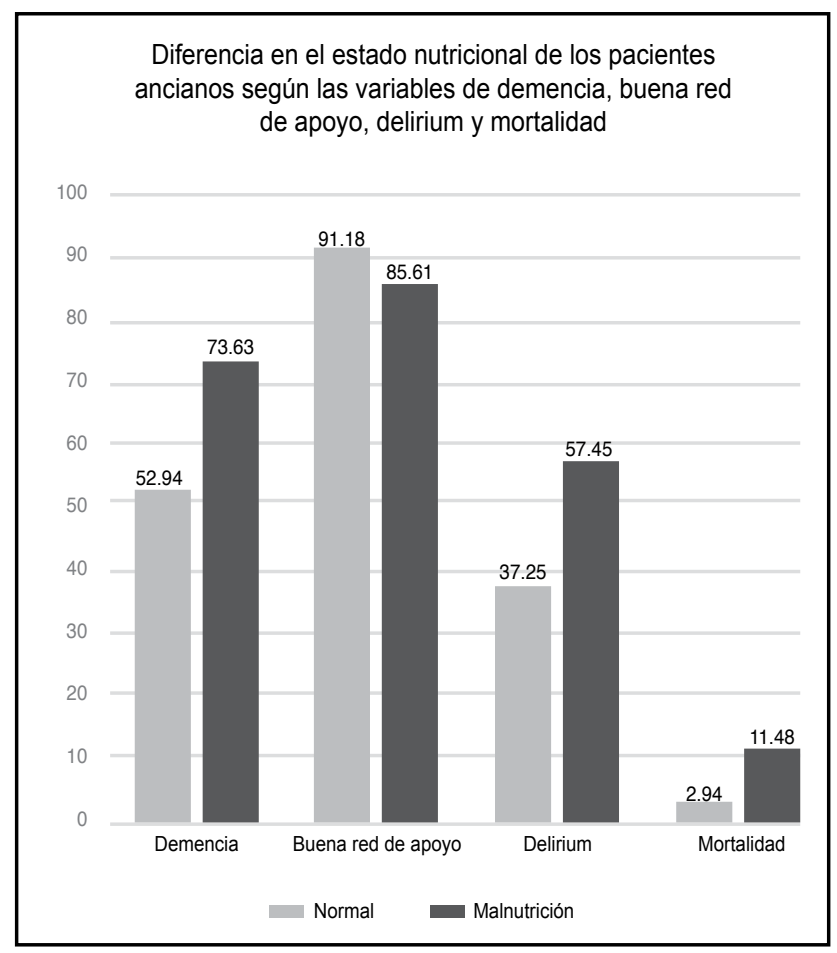

Figura 1. Este gráfico ilustra los porcentajes de las variables cualitativas nominales demencia, delirium y que no egresaron vivos (mortalidad (si)) y una ordinal (buena red de apoyo) según su estado nutricional. El complemento (100 - \#) de los números denotados sobre la figura es el porcentaje de los pacientes que no tuvieron demencia, que no tenían una buena red de apoyo (regular o mala), que no tuvieron delirium o que egresaron vivos (mortalidad (No)). La barra azul representa a los pacientes clasificados con un estado nutricional normal y la naranja como los que están en estado de malnutrición según MNA. Datos tomados de la Tabla 2.

\section{Discusión}

En el estudio se encontró que los pacientes mayores con estado de desnutrición tuvieron una mayor estancia hospitalaria, mayores comorbilidades, así como una menor puntuación en el Barthel al ingreso con respecto a los pacientes clasificados con un estado nutricional normal, lo que coincide con lo encontrado en la literatura $(2,4,6)$.

En adición, la desnutrición y el bajo peso en edades avanzadas se relacionan con deterioro de las funciones cognitivas de los individuos, sarcopenia y atrofia cerebral, resultando en una marcada afectación de la funcionalidad de la persona $(8,9)$. La capacidad funcional de los pacientes estudiados en estado de desnutrición difirió sustancialmente de los que pertenecían al grupo de pacientes con un estado nutricional normal, creando una diferencia de 22.03 puntos en la escala de Barthel. Así, la funcionalidad (Barthel promedio) fue la variable cuantitativa continua con mayor diferencia entre los dos grupos de pacientes estudiados (Figuras 1-2, Tabla 2).

De igual manera, se ha descrito cómo el estado nutricional de las personas mayores influye en la mortalidad de estos mismos al aumentar el número de comorbilidades y sus complicaciones (6). Esto explica la relación estadísticamente significativa que se encontró entre el estado nutricional y la mortalidad de los pacientes mayores (Tablas 2 y 3 ).

Paralelamente, sorprende el hecho que no se haya encontrado una relación estadísticamente significativa entre la desnutrición de los pacientes del estudio y la calidad de la red de apoyo que tenían (OR=1.73 (0.85-3.54); como se encontró en otros estudios $(1,5,7)$. No obstante se observó que tener buena red de apoyo fue la característica cualitativa más común de todo el estudio al presentar una proporción entre 91.18 y $85,61 \%$ en la población con un estado nutricional normal y estado de desnutrición respectivamente (Tabla 3 ).

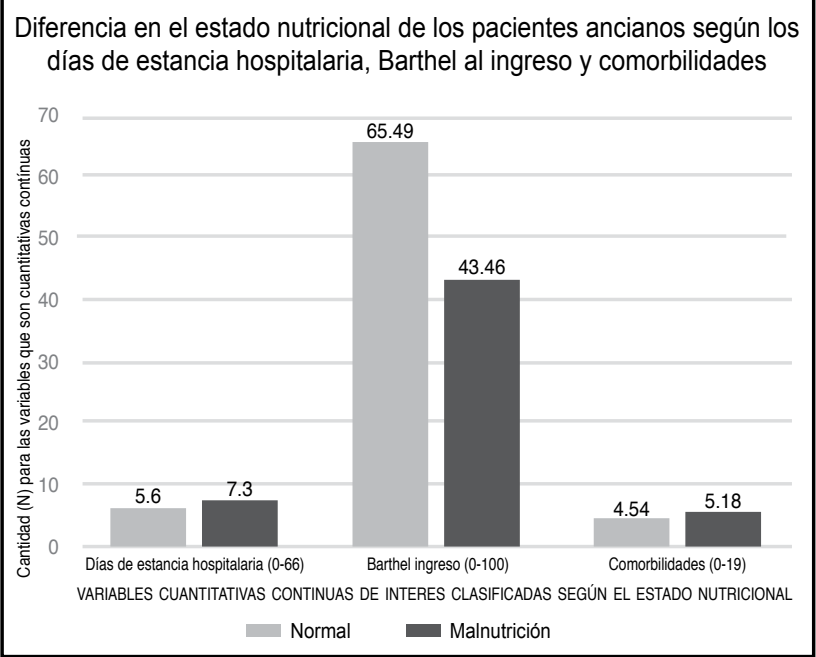

Figura 2. La figura ilustra el promedio de tres variables de carácter cuantitativo continuo según su estado nutricional (azul= normal; -naranja= malnutrición) según el MNA. Datos sacados de la base de datos de geriatría entre 2014 - 2016; organizados en la Tabla 2. 
Otro punto a tener en cuenta es el estado mental de los pacientes, el cual se ha visto relacionado con la elevada prevalencia de la desnutrición en los pacientes mayores. En la literatura se encontró una alta prevalencia de pacientes mayores desnutridos con déficits cognitivos, delirium y demencia (1-5), lo cual explica la asociación estadísticamente significativa entre la presencia de demencia $(\mathrm{OR}=22.48$ (IC $1.63-3.77, \mathrm{p}=<0.001)$ y delirium $(\mathrm{OR}=2.27$, IC 1.48-3.48, $\mathrm{p}=<0.0001)$ en pacientes desnutridos del presente estudio. Sin embargo, es importante resaltar que la prevalencia de demencia en este grupo es superior a la población general (71.25 vs $21.7 \%$ ) (14), puesto que el trabajo fue realizado en un centro reconocido de atención al adulto mayor con demencia.

Cabe resaltar que la única discrepancia en cuanto al valor de $\mathrm{p}$ entre los dos tipos de análisis fue en la variable de egreso vivo-mortalidad, donde en la tabla de significancia estadística (Tabla 2) correspondió a $<0.001$ y en el bivariado (Tabla 3) a 0.015; diferencia que no afecta significativamente el análisis.

Adicionalmente, las características metodológicas de este trabajo no permitieron hacer un análisis prospectivo de la muestra estudiada lo que dificulta inferir una relación causal entre las variables. Así, el tipo de estudio realizado impide obtener información de interés como lo es el aumento de costos hospitalarios a causa de la desnutrición en los pacientes mayores hospitalizados (12).

No obstante, el presente es de los pocos estudios que caracteriza la prevalencia de la malnutrición en pacientes adultos mayores hospitalizados en Bogotá D.C. gracias a sus características metodológicas. Esto puede dar pie a la creación de políticas para el manejo de la población estudiada; un ejemplo sigue siendo uno que haga énfasis en el adecuado soporte nutricional de los hospitalizados teniendo en cuenta que la desnutrición en pacientes mayores acentúa los síntomas y patologías causantes de la hospitalización $(8,9)$.

Finalmente, las características metodológicas de este estudio permiten plantear la creación de nuevas políticas en salud para la atención integral y apropiada de esta población. Se necesitan más estudios que brinden mayor información al respecto de esta problemática

\section{Agradecimientos}

Al Semillero de Neurociencias y Envejecimiento y al Instituto de Envejecimiento de la facultad de medicina de la Pontificia Universidad Javeriana por su incondicional apoyo.

\section{Referencias}

1. Arai K, Sakakibara H. [Malnutrition and social isolation among elderly residents of city public housing]. Nihon Koshu Eisei Zasshi. 2015; 62 (8): 379-389. https:// www.ncbi.nlm.nih.gov/pubmed/26511609. Accessed Mar 7, 2017. doi: 10.11236/ jph.62.8_379.

2. Eide HK, Šaltytė Benth J, Sortland K, Halvorsen K, Almendingen K. Prevalence of nutritional risk in the non-demented hospitalised elderly: A cross-sectional study from norway using stratified sampling. J Nutr Sci. 2015; 4.http://www.ncbi. nlm.nih.gov/pmc/articles/PMC4462759/. Accessed Mar 7, 2017. doi: 10.1017/ jns.2015.8.

3. Rentero Redondo L, Iniesta Navalón C, Gascón Cánovas JJ, Tomás Jimenez C, Sánchez Álvarez C. Malnutrition in the elderly patient to hospital admission, an old problem unsolved. Nutr hosp. 2015; 32 (5): 2169.http://www.ncbi.nlm.nih. gov/pubmed/26545674.

4. Casals C, Vázquez Sánchez MÁ, Casals Sánchez JL, Rioja Vázquez R, Martín Salvador E, García-Agua Soler N. [Relationship between age, body mass index, dependency and quality of life in malnourished patients after hospital discharge]. Nutr Hosp. 2015; 31 (4): 1863-1867. https://www.ncbi.nlm.nih.gov/ pubmed/25795981. Accessed Mar 7, 2017. doi: 10.3305/nh.2015.31.4.8503.

5. Jung Y, Kim J. [Comparison of cognitive levels, nutritional status, depression in the elderly according to living situations]. Taehan Kanho Hakhoe Chi. 2004; 34 (3): 495-503. https://www.ncbi.nlm.nih.gov/pubmed/15314306. Accessed Mar 7 , 2017.

6. Chen L-, Liu L-, Hwang A-, et al. Impact of malnutrition on physical, cognitive function and mortality among older men living in veteran homes by minimum data set: A prospective cohort study in taiwan. J Nutr Health Aging. 2016; 20 (1): 41-47. https://www.ncbi.nlm.nih.gov/pubmed/26728932. Accessed Mar 7, 2017. doi: 10.1007/s12603-015-0646-1.

7. Keller HH, Martin LS, Dupuis S, Reimer H, Genoe R. Strategies to support engagement and continuity of activity during mealtimes for families living with dementia; a qualitative study. BMC Geriatr. 2015; (15): 119.https://www.ncbi. nlm.nih.gov/pubmed/26453451. Accessed Mar 7, 2017. doi: 10.1186/s12877-0150120-2.

8. Vassallo M, Poynter L, Sharma JC, Kwan JSK. Nutritional risk, body mass index and rehabilitation outcome in cognitively impaired vs. cognitively normal patients. Euro Geri Med. 2016; 7 (2): 122-126.http://www.sciencedirect.com/ science/article/pii/S1878764916000371. Accessed Mar 7, 2017. doi: 10.1016/j. eurger.2016.01.010

9. Franco-Álvarez N, Ávila-Funes JA, Ruiz-Arreguí L, Gutiérrez-Robledo LM. Determinants of malnutrition risk among the older adult community: A secondary analysis of the health, wellbeing, and aging study (SABE) in mexico. Rev Panam Salud Púb. 2007; 22 (6): 369-375. http://www.scielosp.org/scielo. php?script=sci_abstract\&pid=S1020-49892007001100001\&lng=en\&nrm=iso\& tlng=es. Accessed Mar 7, 2017. doi: 10.1590/S1020-49892007001100001.

10. Stratégie de prise en charge en cas de dénutrition protéino-énergétique chez la personne âgée. Méd malad métabol. 2007; 1 (4): 92-96. http://www.sciencedirect.com/ science/article/pii/S1957255707741585. doi: 10.1016/S1957-2557(07)74158-5.

11. Giraldo Giraldo NA, Vásquez Velásquez J, Roldán Cano PA, Ospina Astudillo C, Sosa Cardona YP. Cost-effectiveness of early nutritional therapy in malnourished adult patients in a high complexity hospital. Nutri hospi. 2015; 32 (6): 2938. http://www.ncbi.nlm.nih.gov/pubmed/26667756.

12. Vellas B, Guigoz Y, Garry PJ, et al. The mini nutritional assessment (MNA) and its use in grading the nutritional state of elderly patients. Nutrition. 1999; 15 (2): 116-122. https://www.ncbi.nlm.nih.gov/pubmed/9990575. Accessed Mar 16 , 2017.

13. Rehabilitation Section Douglas G. Carroll, Baltimore City Medical Society Editor. Rehabilitation notes.

14. Skoog I, Börjesson-Hanson A, Kern S, et al. Decreasing prevalence of dementia in 85-year olds examined 22 years apart: the influence of education and stroke. Scien Repo. 2017; (7): 6136. doi:10.1038/s41598-017-05022-8. 